\title{
EDITORIAL
}

For reprint orders, please contact: reprints@futuremedicine.com

\section{The role of bevacizumab in recurrent glioblastoma: new insights from randomized trials}

\author{
"The role of bevacizumab in the setting of recurrent glioblastoma is still an \\ argument of debate."
}

Enrico Franceschi' \& Alba A Brandes*,1

The role of bevacizumab in the setting of recurrent glioblastoma (GBM) is still an argument of debate. In Europe, the EMA did not approve this agent despite the promising results in terms of response rate and progression-free survival provided by early Phase II studies without a calibration arm [1,2]. Therefore, new prospective randomized trials with bevacizumab in the recurrent setting have been conducted, and have been recently reported: the BELOB [3] and the AVAREG [4] trials.

Both these trials adopted overall survival rates as primary end points, to avoid issues related to response assessment [5], and included a nitrosourea calibration arm, generating novel information in this setting.

For many years response rate was considered the primary end point for Phase II studies in medical oncology. In neurooncology progression-free survival (PFS) and, in particular the incidence of patients free of progression at 6 months (PFS-6) have been considered the most appropriate end point for Phase II studies since the nineties [6], radiological responses being scarce $(6-8 \%)[7,8]$, and there being a correlation between PFS and overall survival [9].
Yet the action of bevacizumab on blood-brain barrier permeability directly affects the MRI evaluation made in these cases, thus potentially compromising the reliability of the assessment of the response rate and PFS. Therefore PFS-6 was no longer considered a sound end point [5].

In the BELOB trial [3], a randomized Phase II study that enrolled 153 patients to receive bevacizumab alone or in combination with lomustine, or lomustine alone, Taal and Colleagues adopted the Response Assessment in Neuro-Oncology (RANO) criteria [10] for disease assessment, and reported that the radiological response following bevacizumab was correlated with survival (HR: 0.37; 95\% CI: $0.23-0.58)$. These results are consistent with those reported in previous retrospective analyses in which imaging-based end points (response rate or PFS), obtained with bevacizumab were found to correlate with survival [11,12].

Does this suggest that the best end point in Phase II studies should be re-reviewed?

As yet, these findings are suggestive that neuroradiology still has a role in disease assessment for patients treated with bevacizumab, but further data are needed.

'Medical Oncology Department, Bellaria Maggiore Hospitals, Azienda USL - IRCCS Institute of Neurological Sciences,

\section{KEYWORDS}

- bevacizumab - glioblastoma

- survival

"As yet, these findings are suggestive that neuroradiology still has a role in disease assessment for patients treated with bevacizumab, but further data are needed.” 
“... the treatment of glioblastoma is changing, and that future recurrent glioblastoma studies must be undertaken with the utmost caution when evaluating survival time ..."
More interesting data come analyzing survival outcomes across these studies.

In the BELOB study [3] median OS (8 months in each arm) and OS-12 (30 and 26\%), were superimposable between lomustine and bevacizumab as single agents.

The AVAREG trial [4] randomized 91 patients $(2: 1)$ to receive bevacizumab or fotemustine, a third generation nitrosourea, largely used in Italy [13]. RANO criteria were adopted for the disease assessment, and also Macdonald's criteria were recorded. A central review of all the MRI scans was conducted. The primary end point was OS-6, that was 62\% (95\% CI: 48.4-74.5) and 73\% (95\% CI: 54.1-87.7) for bevacizumab and fotemustine, respectively. OS-9 (47 and 38\%), and median OS (8.7 and 7.2 months) were similar between fotemustine and bevacizumab arms.

The superimposable survival results between nitrosoureas and single agent bevacizumab found in both these studies represent a solid and reliable starting point.

For the first time in decades, we have a therapeutic option as effective as standard chemotherapy but with different side effects: mainly hematologic toxicity with nitrosoureas, and cardiovascular with bevacizumab [14]

Thus, patients' selection in recurrent GBM patients will be crucial.

Patients who have experienced hematological toxicities during early treatment phases with temozolomide, and who are likely to have similar toxicities with nitrosoureas could be treated more safely with bevacizumab; while patients with cardiovascular disorders could receive nitrosoureas.

Moreover, in the case of large tumors with edema, bevacizumab may be helpful also for patients' symptoms relief.

These randomized studies highlighted another novel aspect in glioblastoma care, showing that a relevant number of patients received third line treatments (up to $40 \%$ ). Although no prospective data are available on the role of third line treatment for GBM patients, data from Phase III studies may provide us with useful information. In the REGAL trial [15], which compared cediranib, cediranib-lomustine combination and lomustine alone, the lomustine arm had a median survival of 9.8 months, but $60 \%$ of patients were given third line treatments $(51 \%$ bevacizumab). In the NCT00295815 Phase III trial [16], which compared enzastaurin with lomustine, the lomustine arm had a median survival of 7.1 months, while only $10 \%$ of patients were given bevacizumab as third line treatment.

These considerations suggest that the treatment of GBM is changing, and that future recurrent GBM studies must be undertaken with the utmost caution when evaluating survival time, which can be hampered by third line treatments.

The selection of patients and imbalance in prognostic factors remain an issue in Phase II trials: despite randomization, sample sizes are limited.

In the AVAREG trial [4], a numerical imbalance of baseline characteristics in favor of fotemustine in terms of worse PS (19 vs 9\%), corticosteroids use (71 vs 62\%) and tumor area (1024 vs $575 \mathrm{~mm}^{2}$ ) was found, as well a longer time between diagnosis to MRI at screening (331 vs 462 days).

Similarly, in the BELOB trial [3], the time interval between radiotherapy and treatment initiation was longer in patients given lomustine (298 vs 254 days in patients given bevacizumab), the use of corticosteroids lower ( 48 vs $54 \%$ ), the performance status higher (33 vs $26 \%$ ) and the percentage of MGMT methylation and $I D H 1$ mutation $53 \%$ MGMT methylated and $7 \%$ IDH1 mutated) greater than in the bevacizumab arm (43\% MGMT methylated and 3\% IDH1 mutated).

Overall, it is not clear whether these differences may have had an impact on the outcome of patients treated with lomustine, but confirm that results obtained from Phase II should be interpreted with caution.

However, data about bevacizumab in these randomized trials are concordant, suggesting that this agent could be considered another therapeutic option for patients with recurrent GBM.

Financial \& competing interests disclosure The authors have no relevant affiliations or financial involvement with any organization or entity with a financial interest in or financial conflict with the subject matter or materials discussed in the manuscript. This includes employment, consultancies, honoraria, stock ownership or options, expert testimony, grants or patents received or pending, or royalties.

No writing assistance was utilized in the production of this manuscript. 


\section{References}

1 Kreisl TN, Kim L, Moore K et al. Phase II trial of single-agent bevacizumab followed by bevacizumab plus irinotecan at tumor progression in recurrent glioblastoma. J. Clin. Oncol. 27, 740-745 (2008).

2 Friedman HS, Prados MD, Wen PY et al. Bevacizumab alone and in combination with irinotecan in recurrent glioblastoma. J. Clin. Oncol. 27(28), 4733-4740 (2009).

3 Taal W, Oosterkamp HM, Walenkamp AM et al. Single-agent bevacizumab or lomustine versus a combination of bevacizumab plus lomustine in patients with recurrent glioblastoma (BELOB trial): a randomised controlled Phase 2 trial. Lancet Oncol. 15(9), 943-953 (2014).

4 Brandes A, Finocchiaro G, Zagonel V et al. Randomized Phase II trial AVAREG (ML25739) with bevacizumab (BEV) or fotemustine (FTM) in recurrent GBM: final result from the randomized Phase II trial. Ann. Oncol. 25(Suppl. 4), iv137 (2014).

5 Brandes AA, Franceschi E, Gorlia T et al. Appropriate end-points for right results in the age of antiangiogenic agents: future options for Phase II trials in patients with recurrent glioblastoma. Eur. J. Cancer 48(6), 896-903 (2012).
6 Wong ET, Hess KR, Gleason MJ et al. Outcomes and prognostic factors in recurrent glioma patients enrolled onto Phase II clinical trials. J. Clin. Oncol. 17(8), 2572-2578 (1999).

7 Yung WK, Albright RE, Olson J et al. A Phase II study of temozolomide vs. procarbazine in patients with glioblastoma multiforme at first relapse. Br. J. Cancer 83(5), 588-593 (2000).

8 Brada M, Hoang-Xuan K, Rampling R et al. Multicenter Phase II trial of temozolomide in patients with glioblastoma multiforme at first relapse. Ann. Oncol. 12(2), 259-266 (2001).

9 Lamborn KR, Yung WK, Chang SM et al. Progression-free survival: an important end point in evaluating therapy for recurrent high-grade gliomas. Neuro Oncol. 10(2), 162-170 (2008).

10 Wen PY, Macdonald DR, Reardon DA et al. Updated response assessment criteria for high-grade gliomas: response assessment in neuro-oncology working group. J. Clin. Oncol. 28(11), 1963-1972 (2010).

11 Prados M, Cloughesy T, Samant M et al. Response as a predictor of survival in patients with recurrent glioblastoma treated with bevacizumab. Neuro Oncol. 13(1), 143-151 (2011).
12 Han K, Ren M, Wick W et al. Progressionfree survival as a surrogate endpoint for overall survival in glioblastoma: a literaturebased meta-analysis from 91 trials. Neuro Oncol. 16(5), 696-706 (2014).

13 Brandes AA, Tosoni A, Franceschi E et al. Fotemustine as second-line treatment for recurrent or progressive glioblastoma after concomitant and/or adjuvant temozolomide: a Phase II trial of Gruppo Italiano Cooperativo di Neuro-Oncologia (GICNO). Cancer Chemother. Pharmacol. 64(4), 769-775 (2009).

14 Brandes AA, Bartolotti M, Tosoni A, Poggi R, Franceschi E. Practical management of bevacizumab-related toxicities in glioblastoma. Oncologist doi:10.1634/ theoncologist.2014-0330 (2015) (Epub ahead of print).

15 Batchelor TT, Mulholland P, Neyns B et al. Phase III randomized trial comparing the efficacy of cediranib as monotherapy, and in combination with lomustine, versus lomustine alone in patients with recurrent glioblastoma. J. Clin. Oncol. 31(26), 3212-3218 (2013).

16 Wick W, Puduvalli VK, Chamberlain MC et al. Phase III study of enzastaurin compared with lomustine in the treatment of recurrent intracranial glioblastoma. J. Clin. Oncol. 28(7), 1168-1174 (2010). 